\title{
The Role of Mechanoreceptors in and Around the Oral Cavity on Occlusal Function in Overdenture Wearer: A Case Report
}

\author{
Talal H Salame* \\ Department of Prosthodontics, The Lebanes University, Lebanon
}

Received: May 22, 2018; Published: June 07, 2018

*Corresponding author: Talal H Salame, Department of Prosthodontics, Faculty of Dentistry, The Lebanes University, Hadath Campus, Beirut, Lebanon

\begin{abstract}
Motor control alteration of jaw movement after major prosthetic work is a common problem in the field of dental prosthodontics. More teeth are missing in the oral cavity, more complicated is the clinical situation and more requirements are needed for the treatment plan and prosthetic appliances, removable are they or fixed, to achieve stability and satisfaction. In some cases, it could be difficult to achieve acceptable final results following traditional treatment modalities and a deviation from the norms is necessary. This paper discusses a clinical case of removable overdenture adapted with slight modification from the traditional concepts in edentulous patient.
\end{abstract}

Keywords: Removable Prosthodontcis; Dental Attachements, Occlusion; Periodontal Receptors; Mastication

\section{Introduction}

Receptors from different tissues in and around the oral cavity are involved in controlling the spatial position of the lower jaw during function. In dentate patients, mechanoreceptors in the mucosa, periodontal ligament, periostum, muscle spindles and even temporomandibular joint are thought to be involved [1]. At the level of the periodontal ligament, information provided by the mechanoreceptors is transmitted to the Central Nervous System (CNS) where the afferent signals are converted into efferent signals to the muscular motor units that coordinate jaw movement [2]. Patients lacking periodontal ligaments may demonstrate a deviated jaw movement during mastication due to impaired control of the jaw-action vectors [3]. The more limited the periodontal feedback pathways, the more alteration to the fine control of jaw movement [2]. Afferents from other tissues could help compensate by giving positive feedback about the position of the jaw. For instance, in complete denture, pressure of the prosthesis on the underlying mucosa could provoke the mechanoreceptors in the mucosa which can indicate the spatial position of the jaw [4].

In implant patients, the mechanoreceptors from the implant surrounding bone and periostum could play this role [5]. Jaw motor function is possible even with the mouth empty. After the periodontal tissues were anesthetized, subjects were still able to use their occlusal discriminatory ability properly [6]. This suggests that mechanoreceptors from tissues outside the oral cavity could be involved in motor control of the lower jaw. Masticatory muscle mechanoreceptors are possible candidates here. Several studies have demonstrated how muscle spindles and muscle afferents excitation could affect jaw movement and control the spatial position of the jaw $[7,8]$. Other studies emphasized the role of the temporomandibular joint (TMJ) receptors in controlling jaw movement [9-12]. Sensory information from receptors positioned in tissues outside the masticatory system may also help the jaw to control its movement. Signals from visual feedback, for instance, were shown to play an important role in kinesthesia in general [13] and may do so at the level of the masticatory system.

\section{Case Report}

K. S., a 62-year-old female, presented for full mouth prosthetic work. She had an upper removable full denture and a lower removable metallic frame partial denture for 13 years. The patient complained about decreased retention of the lower partial denture and looking older than her age. She requested extraction of her remaining lower teeth and full removable upper and lower dentures. The medical history revealed nothing that could explain the problems or interfere with any dental work. The dental history revealed no previous complications. The patient was satisfied with her dentures until a few years ago. The oral examination showed the upper 
denture was stable on a U shaped well-developed alveolar process and a flat, moderately deep, hard palate. In the lower jaw, the partial denture was class I Kennedy classification (bilateral free ended partially edentulous), with all anterior teeth still in mouth canine to canine. Both right and left alveolar processes were thin, excessively resorbed and highly irretentive and the denture was very unstable. All teeth were vital.

Periodontal examination and X-rays showed calculus deposition on all the lower teeth, with advanced level of recession on 31, 41 and 42, moderate level on 32, and mild on 33 and 43. Palpation revealed class III tooth mobility for 31, 41 and 42, class II for 31, and very mild for the canines. Radiographic examination showed very little bone remaining around the roots of 31, 41 and 42 , and an acceptable level around the root of 32 . The roots of both canines were well preserved with an estimated $19 \mathrm{~mm}$ out of a total root length of $21 \mathrm{~mm}$ surrounded by bone. After discussion with the patient, a decision was made to replace the upper and lower dentures and restore the vertical dimension (VD). For the lower jaw a removable overdenture supported by precision attachments over the two canines was planned. After scaling, teeth 31, 41 and 42 were extracted. Tooth 32 was preserved as support under the denture and to prevent alveolar resorption. Root canal therapies were performed on teeth 32,33 and 43, and two precision ball attachments were adapted over the canines and a cap over 32 .

During manufacture of the dentures, the VD had to be increased by $3-4 \mathrm{~mm}$ for the lower jaw alone to accommodate the two attachment housings under the front teeth. Another $2 \mathrm{~mm}$ had to be added to the vertical dimension using the upper teeth for esthetic purposes. At the trial session, the patient was not comfortable with her new bite as the VD was excessively increased and had to be reduced by $2-3 \mathrm{~mm}$, which would leave no space for setting the lower front teeth above the attachments. During the second manufacture, it proved impossible to set the front teeth above the attachments and decrease the VD at the same time. A decision was made to set the anterior teeth off the alveolar crest anteriorly, and the spaces for the attachment housings were allocated behind rather than under the acrylic front teeth. The second trial was successful, and the VD was restored properly. Both dentures fitted adequately at the delivery session, and the patient was satisfied.

At the follow-up visit one week later the patient complained of discomfort during eating as her front teeth were touching each other. Her speaking was altered when pronouncing some phonemes especially with the letter "s". The clinician gave her gum to chew and observed that the closing phase of her chewing cycle started with the anterior teeth together in an edge to edge position before the mandible slides to the intercarpal position. With a face mirror, she was shown several times how to close her jaw in intercarpal position and was asked to practice repeatedly at home. At the next follow up visit one month later the patient was better adapted to the situation as her speaking and chewing were improved significantly. Subsequently she expressed satisfaction with the results.

\section{Discussion}

It was concluded that this involuntary edge to edge contact of the anterior teeth during function was due to the presence of the roots of the natural teeth supporting the attachments behind the acrylic teeth of the denture. The periodontal receptors can provide important "positive feedback" during biting [14] and play an important role in the control of jaw movement during mastication $[15,16]$. The presence of mechanoreceptors in the periodontal tissues and periostum surrounding the roots of the remaining natural teeth are giving the signal to the lower jaw to occlude with the upper jaw at the level of the precision attachments covering the natural teeth rather than at the level of the artificial teeth set anteriorly. The anteriorly positioned artificial teeth were thus biting on the incisal edges of the upper anterior teeth, giving a faulty impression of an edge to edge occlusion. After the first edge to edge contact, the patient was able to guide her jaw to the required intercarpal position which, in this case, coincided with the centric relation (CR) since the removable prosthesis was a full denture.

This is most likely due to the mechanoreceptors in the TMJ area as they are responsible for the proprioception of mandibular static position and dynamic movement [11]. The CR represents the position of the condyle-disc complex in the glenoid fossa which is a fixed relation with the same bone [12]. When an inadequate occlusal contact occurs, the mechanoreceptors from the TMJ capsule inform the masticatory muscles system to force the mandible to slide until it reaches a comfortable position for the TMJ [12]. It was easier for the patient to adjust her jaw posture with the use of a visual aid. Central control of movement has been modelled as a feedforward mechanism that is modulated by feedback [17]. According to the feedforward model, the set of muscle activations required to execute precise limb movement is defined centrally prior to onset of movement by visual feedback. The feedback system works in modulating the movement when discrepancy between the actual movement and the target occurs [18]. Ferrell et al. [17] developed a model that represents a cut-off between the feedforward and feedback mechanisms.

In this model, "a crude motor plan is assembled prior to the onset of movement and it remains under the constant supervision of a powerful internal feedback loop that adjusts and refines it in real time" [17]. This idea was supported by Milner (1992) who suggested that the CNS sometimes takes action during the movement in order to refine the motor command and bring movement into better alignment [19]. The patient complained of mispronunciation, especially with the letter "s". Speech impairment is a common problem in patients with modified jaw positions [20,21]. In normal conditions, the mandibular movement facilitates speech by sustaining normal tongue movement [20]. An altered mandibular motor pattern due to a modified mandibular posture can affect pronunciation for some letters, especially "s" when followed by the vowel "i" [21]. During further follow-up, all symptoms eventually disappeared, and the patient achieved a stable situation. Long lasting inadequate occlusal contacts can cause modifications in mandibular motor patterns in order for the TMJ and masticatory muscles to adapt to the new mandible-maxilla relation [22]. The perturbation in mandibular movement will overload the masticatory muscles which will react by an adaptive process to keep the system running. This process, defined as "achieving stability through change", explains why symptoms disappear in the majority of patients over time [23]. 


\section{References}

1. Sessle BJ (2006) Mechanisms of oral somatosensory and motor functions and their clinical correlates. J Oral Rehab 33(4): 243-261.

2. Meyer G, Fanghänelb J, ProffbP (2012) Morphofunctional aspects of dental implants. Annals of Anatomy; 194(2): 190-194.

3. Trulsson M, Johansson RS (1996) Forces applied by the incisors and roles of periodontal afferents during food holding and biting tasks. Exp Brain Res 107(3): 486-496.

4. Sakada S (1983) Physiology of mechanical senses of the oral structure. In Kawamura Y, (Eds.). Front. oral physiol Karger, Basel, Switzerland, 14: pp. 1-32.

5. Enkling N, Heussner S, Nicolay C, Bayer S, Mericske-Stern R, et al. (2012) Tactile sensibility of single-tooth implants and natural teeth under local anesthesia of the natural antagonistic teeth. Clin Implant Dent R 14(2): 273-280.

6. Riis D, Gidden, DB (1970) Interdental discrimination of small thickness differences. J Prosthetics Dent 24(3): 324-334.

7. Jacobs R, Steenberg he D Van (1994) Role of periodontal ligament receptors in the tactile function of teeth: a review. J Periodont Res 29(3): 153-167.

8. Ostry DJ, Vatikiotis Bateson E, Gribble PL (1997) An examination of the degrees of freedom of human jaw motion in speech. J Speech Lang Hear Res 40(6): 1341-1351.

9. Yoshida K (1998) Masticatory muscle responses associated with unloading of biting force during food crushing. J Oral Rehabil 25(11): 830-837.

10. Caffesse RG, Carraro JJ, AlbanoEA(1973) Influence of temporomandibular joint receptors on tactile occlusal perception. J Periodontal Res 8(6): 400-403.

11. Clark RKF, Wyke BD (1974) Contributions of temporomandibular articular mechanoreceptors to the control of mandibular posture: an experimental study. J Dent 2(3): 121-129.

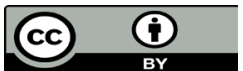

This work is licensed under Creative Commons Attribution 4.0 License

Submission Link: https://biomedres.us/submit-manuscript.php
12. Douglas CR, Avoglio JLV, de Oliveira H (2010) Stomatognathic adaptive motor syndrome is the correct diagnosis for temporomandibular disorders. Med Hypotheses 74(4): 710-718.

13. Coello Y (2005) Spatial context and visual perception for action. Psicológica 26: 39-59.

14. Lund J P, Lamarre Y (1973) The importance of positive feedback from periodontal pressoreceptors during voluntary isometric contraction of jaw closing muscles in man. J Biol Buccale 1(4): 345-351.

15. Trulsson M, Johansson RS, Osson KA (1992) Directional sensitivity of human periodontal mechanoreceptive afferents to forces applied to the teeth. J Physiol 447: 373-389.

16. Trulsson M, Gunne HSJ (1998) Food-holding and -biting behavior in subjects lacking periodontal receptors. J Dent Res 77:574-582.

17. Ferrell WR, Gandevia SC, McCloskey DI (1987) The role of joint receptors in human kinaesthesia when intramuscular receptors cannot contribute. J Physiol 386: 63-71.

18. Desmurget M, Grafton S (2000) Forward modeling allows feedback control for fast reaching movements. Trends in Cogn Sci 4(11): 423-431.

19. Milner TE (1992) A model for the generation of movements requiring end point precision. Neurosci Res 49(2): 487-496.

20. Ostry DJ, Munhall KG (1994) Control of Jaw Orientation and Position in Mastication and Speech. J neurophysiol 71(4): 1528-1545.

21. Smith A (1992) The control of orofacial movements in speech. Crit Rev Oral Biol M 3(3): 233-267.

22. Areso MP, Giralt MT, Sainz B, Prieto M, GarcíaVallejo P, et al. (1999) Occlusal disharmonies modulate central catecholaminergic activity in the rat. J Dent Res 78(6): 1204-1213.

23. McEwen BS, Wingfield JC (2003) The concept of allostasis in biology and biomedicine. HormBehav 43(1): 2-15.

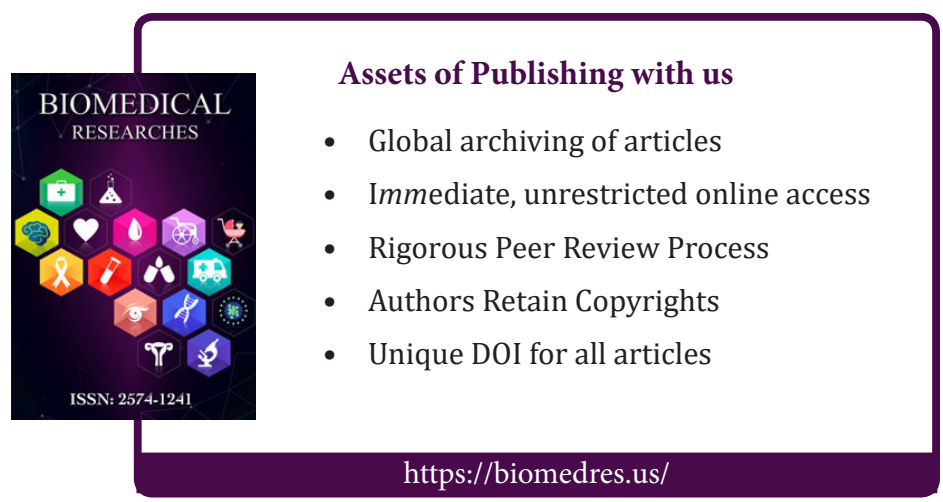

